\title{
Dynamic design of automotive systems: Engine mounts and structural joints
}

\author{
R SINGH \\ Department of Mechanical Engineering, Ohio State University, Columbus, \\ $\mathrm{OH}$ 43210, USA \\ e-mail: singh.3@osu.edu
}

\begin{abstract}
Dynamic design and vibro-acoustic modelling issues for automotive structures are illustrated via two case studies. The first case examines the role and performance of passive and adaptive hydraulic engine mounts. In the second, the importance of welded joints and adhesives in vehicle bodies and chassis structures is highlighted via generic ' $T$ ' and ' $\mathrm{L}$ ' beam assemblies. In each case, analytical and experimental results are presented. Unresolved research issues are briefly discussed.
\end{abstract}

Keywords. Dynamic design; engine mounts; structure joints; automotive systems; vibro-acoustic modelling.

\section{Introduction}

An automotive engine-body-chassis system is typically subjected to unbalanced engine forces, uneven firing forces especially at the idling speeds, dynamic excitations from gearboxes and accessories, and road excitation. Since design trends have been towards compact and efficient automobiles, engine-to-frame weight ratio and engine force densities have increased. Consequently, recent research and development efforts have been focused on improving engine mounting technology to achieve better vibration isolation, smooth vehicle movement, and noise reduction. Such dynamic design issues are illustrated via the hydraulic engine mount case study. Results of mathematical models and experimental studies are presented, and limitations of the passive device are briefly discussed. A new adaptive engine mounting system is proposed, and comparative results are briefly examined.

The dynamic behaviour of welded joints, mechanical fasteners and adhesives in thin sheet automotive structures is poorly understood since engineering design data such as bending or torsional stiffnesses and damping loss factors are virtually nonexistent. Consequently, current design practice is largely empirical, based mostly on the designer's experience and intuition. Design requirements for static and dynamic loadings may conflict, as low stresses in joints demand rigid connections, while designs for low vibration and noise often require more compliant joints. In practice, however, no analytical design tools are available to resolve such issues. While finite element (FEM) solutions are useful if 
properly implemented, a separate problem-specific solution must be constructed for each joint/structure problem considered. A more general set of analysis tools and design dynamics is required to understand existing designs better and to develop alternate joints based on new or improved welding processes. A problem is formulated in this article with ' $\mathrm{T}$ ' and ' $\mathrm{L}$ ' beam assemblies as generic examples. Procedures for determining joint stiffness are discussed.

\section{Case study A: Hydraulic engine mounts}

\subsection{Passive mount concept}

An engine mount must satisfy two essential but conflicting criteria. First, it should be stiff and highly damped to control the idle shake and engine mounting resonance over $5-30 \mathrm{~Hz}$. Also, it must be able to control, like a shock absorber, the motion resulting from quasistatic load conditions such as travel on bumpy roads, abrupt vehicle acceleration or deceleration, and braking and cornering. Second, for a small amplitude excitation over the higher frequency range, a compliant but lightly damped mount is required for vibration isolation and acoustic comfort, like a conventional rubber mount. A conventional rubber mount cannot satisfy both requirements simultaneously as the lumped stiffness $k_{r}$ and the viscous damping coefficient $b_{r}$ in the shear mode are nearly invariant with excitation amplitude and frequency over the concerned excitation range (say $1-250 \mathrm{~Hz}$ ) of vehicle systems. Thus, a compromise between resonance control and isolation is inevitably needed. The mount is typically optimized for placement, orientation, $k_{r}$ and $b_{r}$. To meet both performance requirements, hydro-mechanical mounts have been designed recently and employed in many vehicles. Figure 1 shows a schematic representation. Such a mount can provide improved stiffness and damping characteristics which vary with frequency and excitation amplitude. It is conceptually the best passive engine mount known at present.

\subsection{Mathematical models of passive mount}

Typical dynamic properties are illustrated in figure 2 where $K$ is the dynamic stiffness magnitude, $\phi_{k}$ is the loss angle and $X$ is the displacement amplitude. Analytical predictions are also compared in this figure based on a nonlinear model that is described in several articles (Singh et al 1992; Kim \& Singh 1993, 1995). Excellent agreement between theory and experiment is seen up to $50 \mathrm{~Hz}$.

Figure 3 shows a conceptual quarter-car model that includes the nonlinear mounts and linear suspension system. Here $k_{r}$ and $b_{r}$ are frequency variant stiffness and damping coefficients of the top rubber element (in shear mode), $m_{e}$ and $m_{s}$ are engine mass and vehicle mass respectively, and $u(\omega, x)$ is the time-varying nonlinear hydraulic reaction force. Figure 4 compares the sprung mass acceleration amplitude $\ddot{X}_{s}(\omega)$ spectra for the rubber mount and hydraulic mounts with decoupler gap $\Delta_{d}=0,0.7$ and $1.4 \mathrm{~mm}$. A high resonant peak occurs at $9.2 \mathrm{~Hz}$ for the low damping rubber mount. The inertia track hydraulic mount with $\Delta_{d}=0$ clearly shows its superior dynamic performance up to $15 \mathrm{~Hz}$. Nonlinear effects are seen for $\Delta_{d}=0.7$ and $1.4 \mathrm{~mm}$ when both inertia track and decoupler mechanisms are employed. Limitations of the inertia track mount may be seen beyond $15 \mathrm{~Hz}$ since it yields high mount stiffness vibration and higher transmissibility. One may 


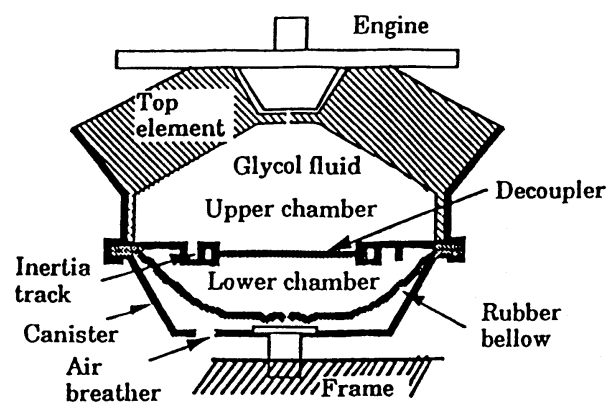

Figure 1. Hydraulic engine mount (passive design).
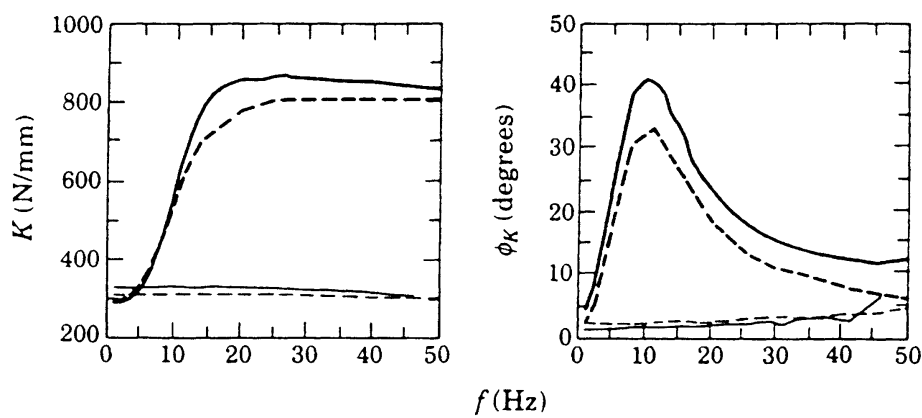

Figure 2. Typical dynamic characteristics of hydraulic mount. Predicted and measured dynamic stiffness spectra of the regular mount. (--- Theory for $X=0.1 \mathrm{~mm}:-$ experiment for $X=0.1 \mathrm{~mm}$; -- theory for $X=1.0 \mathrm{~mm}$ : experiment for $X=1.0 \mathrm{~mm}$.)

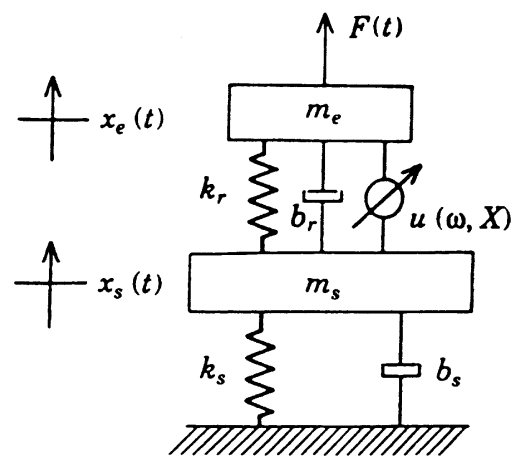

Figure 3. Quarter-car model with nonlinear hydraulic mount.

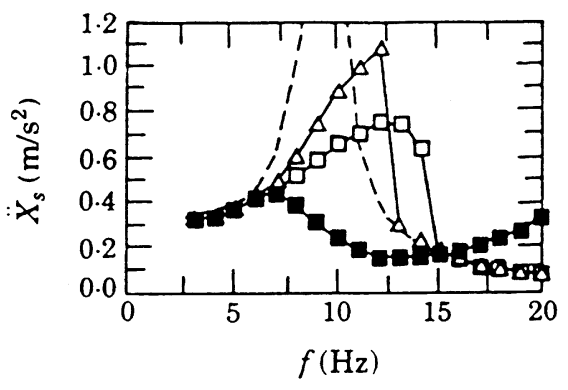

Figure 4. Comparison of rubber and hydraulic mounts using quarter-car model. Simulated harmonic responses of the vehicle model for $F_{a}=100 \mathrm{~N}$. (Effect of decoupler gap on $\ddot{X}_{s}(\omega): \mathbf{\square}, \Delta_{d}=0 \mathrm{~mm}: \square, \Delta_{d}=0.7 \mathrm{~mm}$ : $\triangle, \Delta_{d}=1.4 \mathrm{~mm}:---$ rubber mount.) 
indeed prefer a rubber mount at higher frequencies. Further information on nonlinear models of hydraulic mounts and vibratory power flow concepts may be found in the literature (Singh et al 1992; Kim \& Singh 1993, 1995, Roystan \& Singh 1995-1997).

\subsection{Performance limitations}

Given the results of the preceding sections, a few conclusions can be drawn regarding the performance limitations of various passive mount configurations. Specifically, the inherent nature of the regular hydraulic mount is that engine resonance around $10 \mathrm{~Hz}$ must be initiated before the mount is able to dissipate any engine mounting vibratory energy. In other words, fluid damping related to the inertia track flow cannot be generated during the relative engine motion corresponding to the decoupler gap. Yet, on the other hand, other limitations are associated with the undesirable side-effects of fluid inertia. For instance, noise, vibration and harshness $(\mathrm{NVH})$ problems of the regular mount result from fluid resonances at frequencies beyond $100 \mathrm{~Hz}$. Furthermore, the fluid inertial effect influences the vibration isolation properties of the regular mount even around $20 \mathrm{~Hz}$. To overcome such problems, a new adaptive mount is proposed.

\subsection{A new adaptive hydraulic mount}

A low damping rubber mount is most suitable for isolating the engine disturbance force from the vehicle frame at frequencies beyond the engine mounting resonance. On the other hand, the inertia track mount is most appropriate in controlling engine resonance amplitude and absorbing the shock excitations. Accordingly, the basic premise behind the proposed broadband adaptive system is to let the hydraulic mount function as a rubber mount for the purpose of vibration and acoustic isolation, and as an inertia track mount for resonance control and shock absorption. This adaptive system comprises two modules: a mechanical actuation system and an electronic controller. As shown in figure 5, the adaptive system can operate in either a "hard" or "soft" state. The rubber sheet, installed just under the top element, and the lower rubber bellow are connected to an engine intake-manifold vacuum through two-position, three-way on-off solenoid valves which are controlled by an ECM. Since intake-manifold vacuum reaches down to $27-44 \mathrm{kPa}$ (absolute) or $17-22$ in $\mathrm{Hg}$ vacuum during the normal vehicle operation, it may serve as the vacuum source for our adaptive system. Otherwise, a small vacuum pump may be employed, especially for a diesel engine where there may not be any vacuum source. The operating principles of the adaptive systems are as follows.

Hard state (figure 5a)-If high damping is needed for resonance control and shock absorption, a vacuum pressure is applied to the upper rubber sheet through valve 1 while the lower bellow is open to the atmosphere through valve 2. Fluid mass is pulled up and the upper rubber sheet is coupled to the top element. As a result, the adaptive mount basically becomes an inertia track hydraulic mount.

Soft state (figure 5b) - If a low dynamic stiffness and damping property is desirable for the vibration isolation, vacuum is applied directly below the lower bellow through valve 2 while the upper rubber sheet is open to the atmosphere through valve 1. Fluid mass is pulled down and the upper rubber sheet is decoupled from the top element. As a result, the 


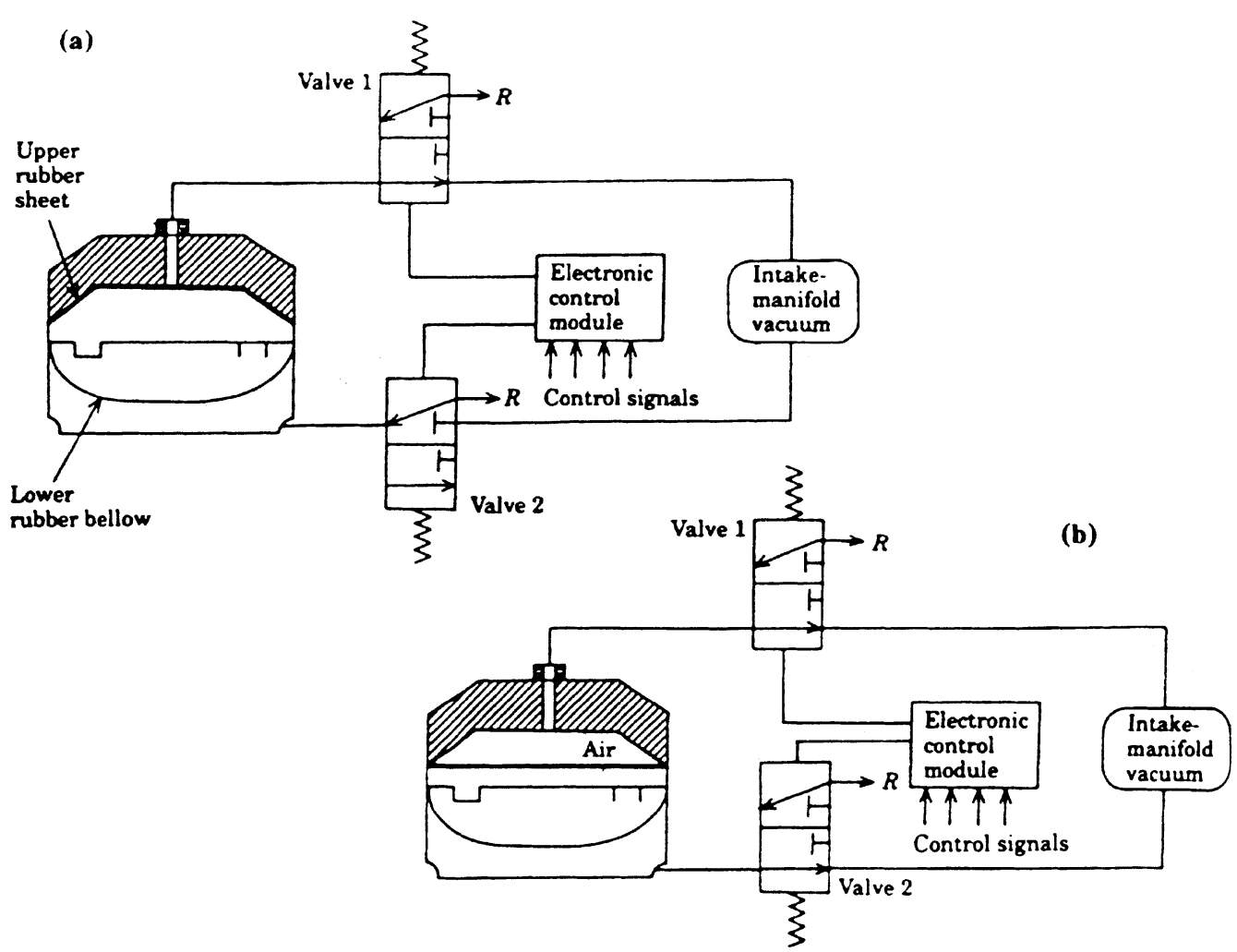

Figure 5. The operation of the proposed adaptive hydraulic mount. Hard, (a) and soft (b) states.

engine motion cannot excite fluid vibration, and the adaptive mount essentially becomes a low-damping rubber mount.

Figure 6a compares passive, adaptive and active mount concepts over the low frequency regime. Figure $6 \mathrm{~b}$ compares the spring mass accelerance $\alpha_{s}$ in the frequency range 50 to $250 \mathrm{~Hz}$ where $K$ of the engine mount is measured with $X=0.1 \mathrm{~mm}$. The adaptive mount, functioning in the soft state, yields superior vibration isolation characteristics over the
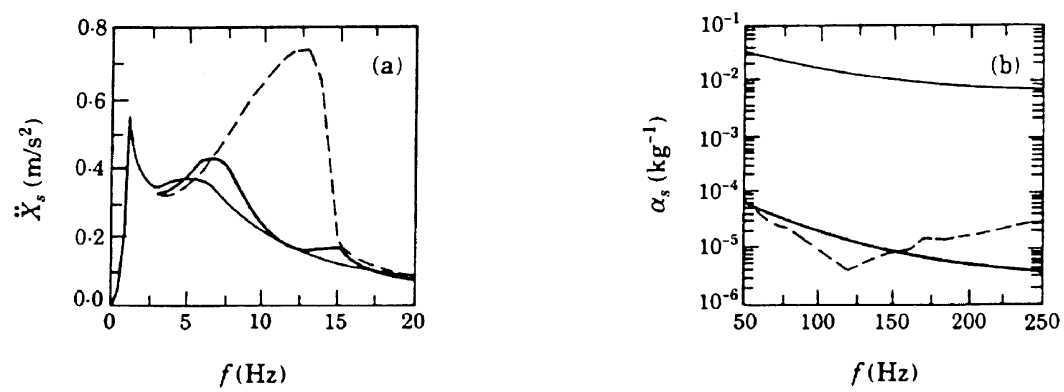

Figure 6. Comparison of passive, adaptive and active mounts. Harmonic responses for three mounting modes in the vehicle model: - - passive mount; - adaptive mount; active mount. (a) $\ddot{X} .(\omega)$ at lower frequencies: $(\mathbf{b}) \alpha_{s}(\omega)$ at higher frequencies. 
passive mount. In actual vehicle testing, it has been observed that the passive mount causes high "boom" noise levels. Consequently, figure 6 illustrates that the adaptive engine mount exhibits a sufficiently low dynamic stiffness at higher frequencies, which should control the booming noise. Observe that in addition to yielding excellent harmonic responses, the adaptive mount should improve both ride quality and vehicle durability for the shock excitation during an engine load change. Note that the actual transient response will depend on the switchover response speed of the adaptive mount.

\subsection{Research issues}

Further research is, however, needed to develop high frequency models, as well as for an improved understanding of the nonlinear characteristics. Second, conceptual development and hardware implementation have been presented for a new broadband adaptive hydraulic mount system, which employs a vacuum pressure already existing in the engine intake manifold, and two solenoid valves. Even though technical prospects and practical aspects appear promising, the actual performance must still be examined through vehicle tests. Related research objectives which need to be addressed in a future study are as follows. (i) To determine the threshold engine speed at which the switching between the hard and soft states takes place, and its dependence on vehicle conditions, i.e., specific algorithms for switchover conditions; (ii) where and how to install the adaptive mount under the engine transmission block; and (iii) whether the engine intake-manifold vacuum system needs to be boosted, since a loss of intake-manifold vacuum occurs during vehicle acceleration or climbing.

\section{Case study B: Discrete joints in thin sheet metal structures}

\subsection{Problem formulation}

While many joints and machine elements are inherently nonlinear, only linear timeinvariant characteristics over the lower frequency regime are considered here as a logical first step (Singh et al 1995; Farstad \& Singh 1995, 1996; Rook \& Singh 1995-1997). As a consequence, mechanical fasteners such as bolted connections are not considered at this stage. Yet another important research issue is the dimension of the joint. An example of this is the situation in which both the force and moment transmissions through a joint are significant. Although moment paths may be negligible at lower frequencies, some studies suggest otherwise. For the sake of simplifying formulations, several studies have used scalar joints, but in the case of more general joints, vector transmissions paths must be considered.

Several existing narrow-band analysis methods are in the form of component synthesis procedures, thereby allowing better examination of the consequences of design modifications to a particular substructure or component. Most of these procedures synthesize components in either the modal or the frequency domain. Since none of the existing methods is believed to be fully capable of addressing the issues associated with multi-dimensional compliant joints, we have chosen to extend and refine two established narrow band analysis procedures. One method is based on a mobility approach (Rook \& Singh 1995, 1996). The other is based on a modal synthesis approach where proportional damping is assumed such that all the modes are real-valued; this particular method is 
discussed by Farstad \& Singh $(1995,1996)$. Only a subset of the comprehensive project is presented in the current article. In the overall study, the assembled structure is decomposed into three key components, in the form of a "classical" noise and vibration control problem, for the analysis including free and force vibration responses along with power flow calculation. The source structure contains the origin of the narrow-band vibrational energy (excitation), and is the only active component. The next component represents the massless, non-conservative, and non-rigid joints which may be multi-dimensional and connect the adjoining structures at several discrete locations. From the joints, the power is then transmitted to the passive receiver structure (c), which for the sake of convenience can be further decomposed into free receiver and foundation components. Often, the foundation component may be modelled as a generalized termination impedance or energy sink. The knowledge of modal base is essential to the calculation of dynamic power (Farstad \& Singh 1995, 1996; Rook \& Singh 1995, 1996).

\subsection{T-beam structure with an ideal joint}

Consider a T-shaped assembly of beams, as shown in figure 7a. A new formulation, based on a component modal synthesis approach as developed (Farstad \& Singh 1995, 1996; Rook \& Singh 1995, 1996), was applied to this problem to compute the vibration transmitted through the joint. Natural frequencies and modes of the assembly are obtained from those of its components by using variational methods. The constraints that motions of components be identical at the joint locations are enforced using Lagrange multipliers, which are equal to the interfacial forces and moments. Evaluating them from the forced response of the assembly provides an efficient means for computing the mechanical power transmitted through various structural paths, which is the best measure of transmitted vibration. This assembly is considered as consisting of three components, as indicated in figure $7 \mathrm{~b}$. The formulation requires that natural frequencies and modes of individual components corresponding to fixed conditions imposed at the joint location be known. The flexural and longitudinal motions of the assembly are coupled, so all individual components are straight beams, however, the component modes correspond either to purely flexural or purely longitudinal motion.

The new formulation for modal synthesis and transmitted vibration is applied to determine modal properties, forced response, and vibration transmission characteristics of the long T-beam assembly. The length of each component beam was taken to be $L_{1}=L_{2}=L_{3}=254 \mathrm{~mm}$, and each was considered to be $3.2 \mathrm{~mm}$ thick, $25.4 \mathrm{~mm}$ wide, and made of carbon steel with properties $E=207 \times 10^{9} \mathrm{~N} / \mathrm{m}^{2}$ and $p=7832 \mathrm{~kg} / \mathrm{m}^{3}$. The
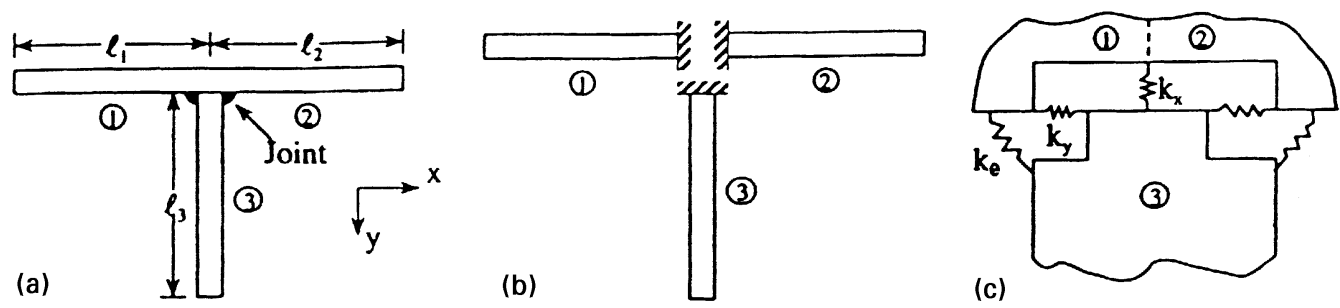

Figure 7. "T" beam assembly and synthesis approach. (a) Schematic, (b) component synthesis for an ideal joint, (c) joint stiffness matrix model. 
Table 1. Natural frequencies of the long ' $\mathrm{T}$ '-beam assembly for the ideal joint.

\begin{tabular}{lcccccccc}
\hline & \multicolumn{7}{c}{ Natural frequency $(\mathrm{Hz})$ at mode } \\
\cline { 2 - 9 } Method & 1 & 2 & 3 & 4 & 5 & 6 & 7 & 8 \\
\hline Experiment & 42.5 & 50.5 & 169 & 255 & 275 & 544 & 701 & 720 \\
Theory & 42.2 & 50.5 & 172 & 251 & 275 & 555 & 690 & 717 \\
\hline
\end{tabular}

assembly natural frequencies obtained from the analysis appear in table 1 together with those obtained from measurements on a welded T-beam assembly of the same material and dimensions. Agreement between predicted and measured natural frequencies is excellent, with maximum differences being less than two percent. The forced response of the assembly was then computed for a concentrated force of $1 \mathrm{~N}$ magnitude and uniform harmonic content acting at the free end of the shorter beam. Uniform damping ratios $\zeta=0.002$ were applied to all assembly modes. The dynamic compliance magnitude at the drive point for excitation in the $x$-direction appears in figure 8 together with the corresponding measured result. The predicted and measured results agree quite well.

\subsection{Determination of joint stiffnesses}

Figure 9 shows a flow chart which is used to determine the joint stiffness in components when an unknown joint is encountered. The basic concept for obtaining the joint stiffnesses in the T-beam assembly is as follows. At low frequencies, the natural frequencies of symmetric modes depend on the stiffness of the $y$ direction $\left(k_{y}\right)$ and a symmetric mode depends on the stiffness in $x$ and $\theta$ directions $\left(k_{x}, k_{\theta}\right)$, where $k_{x}, k_{y}$, and $k_{\theta}$ are elements of the joint stiffness matrix $\left[k_{i j}\right]$; note that a $2-\mathrm{D}$ representation is sufficient for this problem.

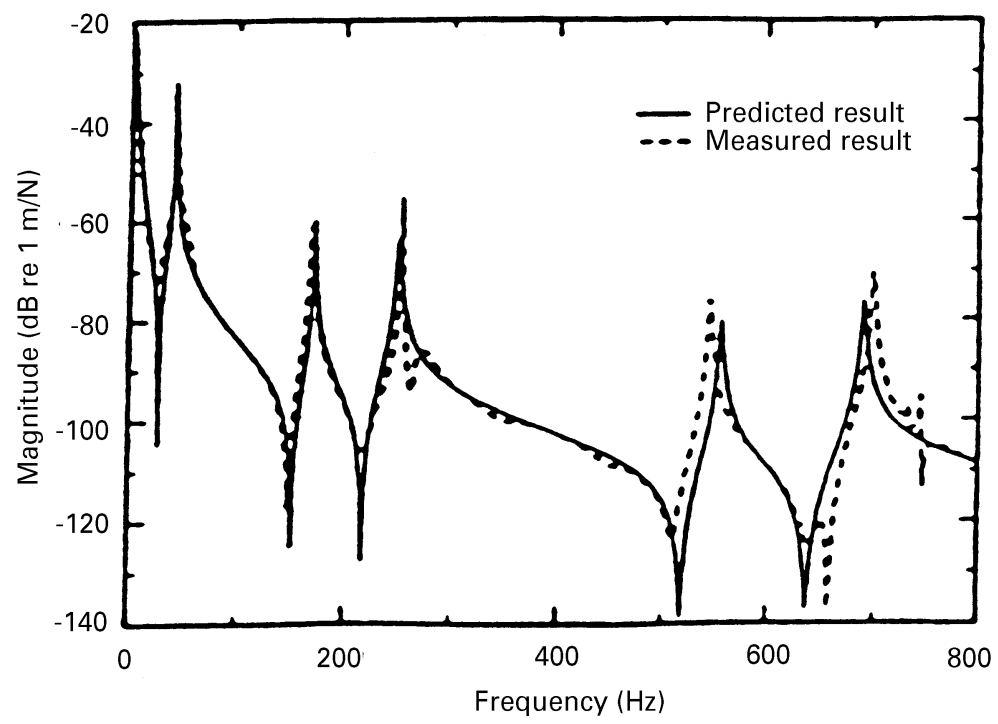

Figure 8. Driving point compliance for " $\mathrm{T}$ " beam. 


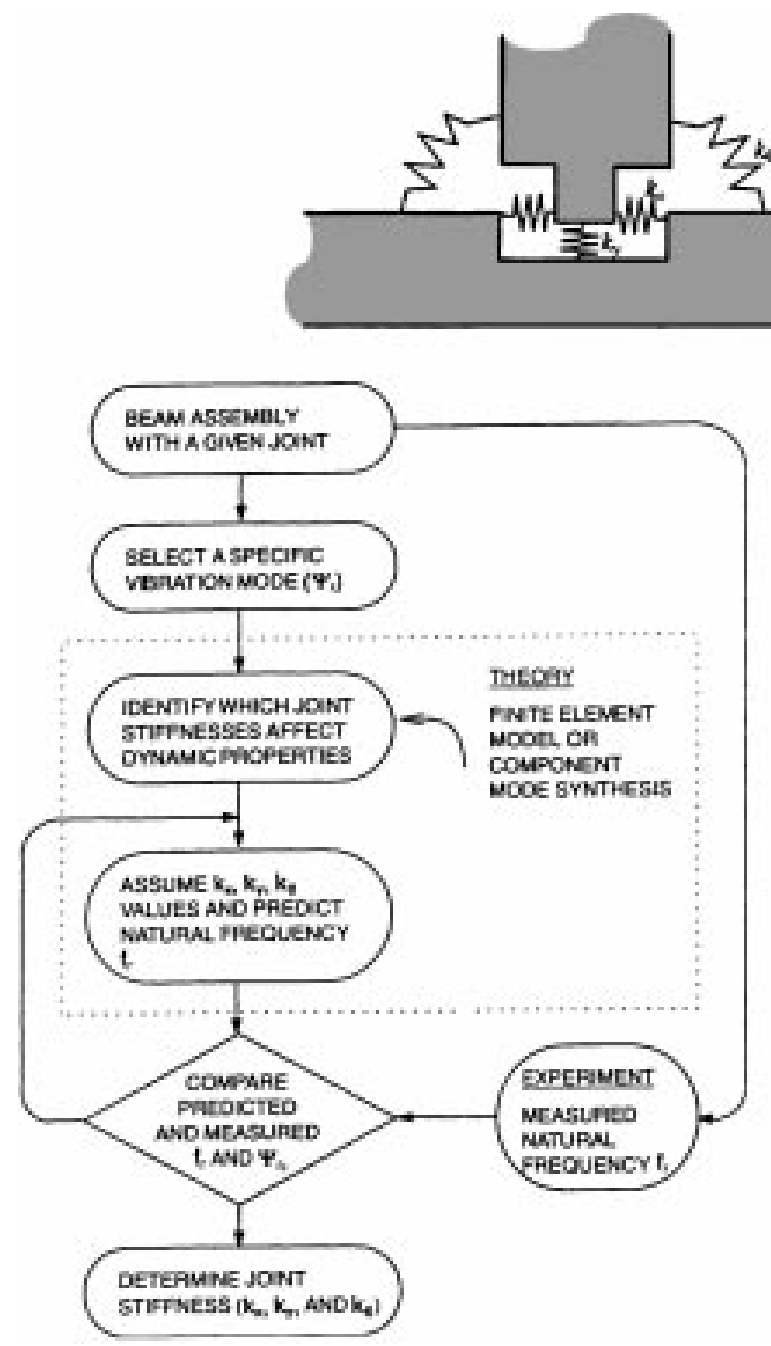

Figure 9. Procedure for estimating the joint stiffness matrix.

Such relationships between natural frequencies and the joint stiffnesses have been determined for the $\mathrm{T}$ structure. The natural frequency of the selected mode can easily be found from experiment. Figure 10 shows a somewhat generic relationship. This information can be used for design as well as for diagnostic purposes.

Observe in figure 10 that there are three distinct regimes in natural frequency vs joint stiffness curves. When the joint stiffness is very small, the natural frequencies of the coupled structure converge to the natural frequencies of the individual beam components (region I). When the joint stiffness is very large, the natural frequencies are essentially the frequencies of the components that are connected rigidly at the joint (region III). There is a transaction regime (II) between these two extreme stiffness values. In this regime, the effects of joint compliance are most significant. Several experiments were designed to verify the existence of three regimes and some results are reported here. 

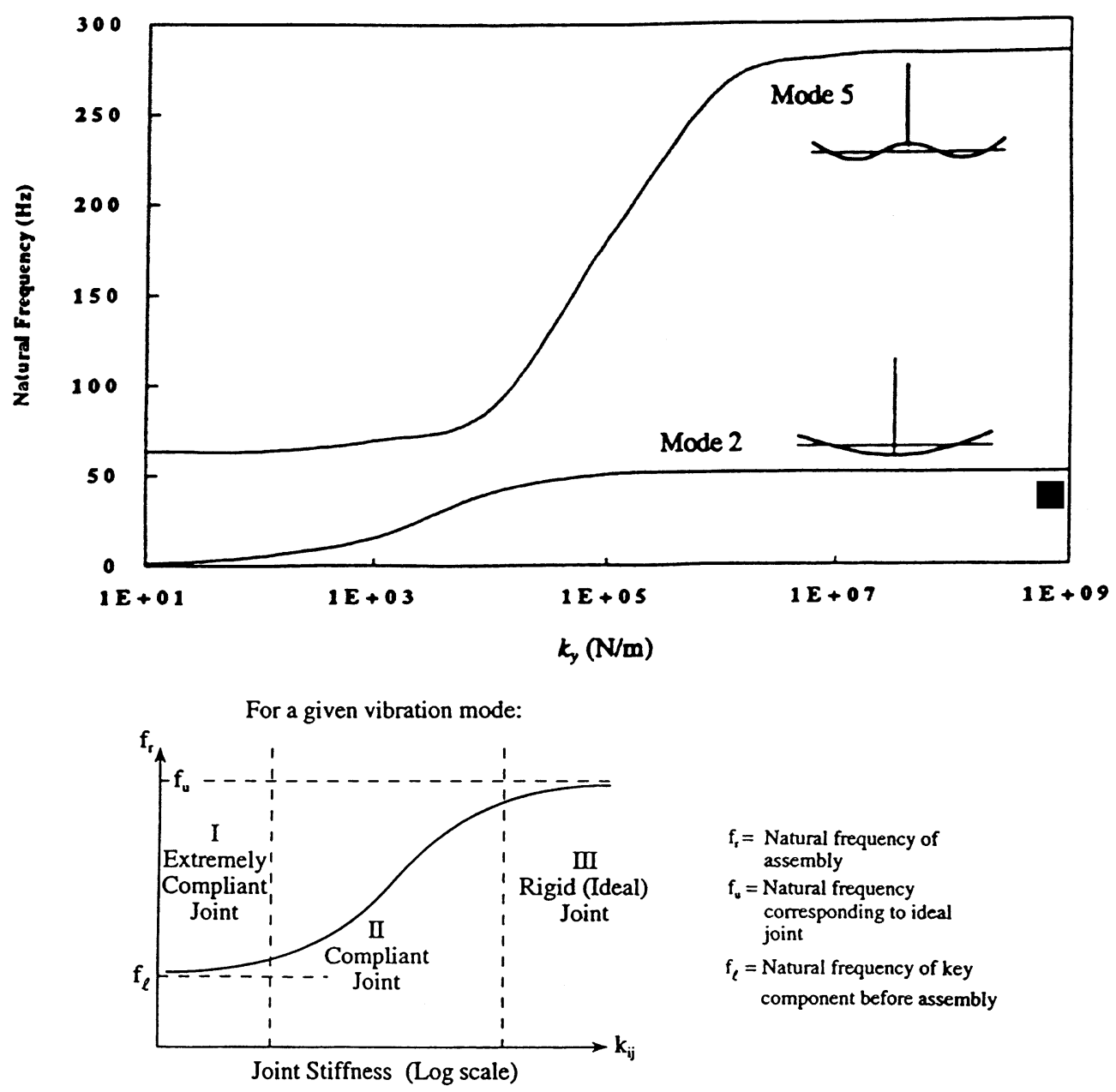

Figure 10. Natural frequency versus joint stiffness relationship depicting three distinct regimes.

\subsection{Results on ' $T$ ' and ' $L$ ' beam structures with alternate joints}

The first experiment was designed to verify the high stiffness regime. After finding the natural frequencies, the procedure of figure 9 is applied to find the stiffness values. These values were then used in a finite element model of the assembly to verify the component mode synthesis procedure discussed earlier. A comparison between all methods including the modal experiment is given in table 2 . The dimensions of the short T-beam steel structure are: $L_{1}=L_{2}=76.2 \mathrm{~mm}, L_{3}=127 \mathrm{~mm}$ thickness $=3.175 \mathrm{~mm}$, and width $=12.7 \mathrm{~mm}$. The results show that the calculated natural frequencies match the measured natural frequencies very well. However, several modelling possibilities or schemes exist when employing synthesis or FEM procedures, hence there is a variation in predictions. This case suggests that the component mode synthesis methods work well when the joint stiffness is indeed very large, i.e. it can be modelled as a rigid joint. Also, a nearly complete set of component modes must be included, otherwise modal truncation errors are seen. 
Table 2. Natural frequencies of the short ' $\mathrm{T}$ '-beam assembly with alternate joints.

\begin{tabular}{llccc}
\hline & & \multicolumn{3}{c}{ Natural Frequency $(\mathrm{Hz})$} \\
\cline { 3 - 5 } Joint type & Method & Mode 1 & Mode 2 & Mode 3 \\
\hline Welded joint & Experiment & 184 & 560 & 832 \\
& FEM & $181-187$ & $548-562$ & $831-856$ \\
& Theory & $173-186$ & $551-722$ & $785-833$ \\
Epoxy glue & Experiment & 144 & 520 & 795 \\
& Theory & 207 & 516 & 799 \\
Silicon glue & Experiment & 179 & 683 & 1010 \\
& Theory & 180 & 688 & 1083 \\
\hline
\end{tabular}

The next series of physical experiments was designed to investigate the low stiffness regime. The procedure of figure 9 is used again to determine $k_{x}, k_{y}$ and $k_{\theta}$. It is observed that the silicon glue joint is a very compliant or weak joint, however the epoxy joint is found to be stiffer than expected. Table 2 also lists results for such adhesive joints. It is seen that the component mode synthesis method can estimate the first two natural frequencies for silicon joint type accurately. However, there is a larger error in the first mode of epoxy joint. Also note that the mass of the accelerometer has a significant effect on the measured natural frequencies, which introduces an error when the experimental values for thin compliant structures are utilized to estimate stiffness at the joint. In this case, there are still some modes that cannot be predicted by the beam model which implies that the current model needs to be modified.

Many other beam and plate assemblies have been examined or are being studied. One example is the ' $\mathrm{L}$ ' beam assembly which can be viewed as a case of ' $\mathrm{T}$ ' structure of figure 5 a where $L_{2}=0$. Other dimensions are: $L_{1}=L_{3}=254 \mathrm{~mm}$, thickness $=1.52 \mathrm{~mm}$ and width $=25.4 \mathrm{~mm}$. Sample results are given in table 3 .

\subsection{Research issues}

An analytical framework has been developed which utilizes the joint stiffness and modal database of sub-structures to calculate the vibration of the assembled sheet metal structure. This analysis method is essentially the component mode synthesis method. Experiments have been performed on the ' $\mathrm{T}$ ' and ' $\mathrm{L}$ ' beam structures to verify the theory developed and to determine additional joint properties such as the mechanical damping of common joints. Damping information from the experiments can be used to extend the joint stiffness matrices to include damping and improve the theory as necessary.

Table 3. Natural frequencies of the ' $L$ ' beam assembly with alternate joints.

\begin{tabular}{lcccc}
\hline & & \multicolumn{3}{c}{ Natural frequency $(\mathrm{Hz})$} \\
\cline { 3 - 5 } Joint & Method & Mode 1 & Mode 2 & Mode 3 \\
\hline Rivet & & 21 & 83 & 116 \\
Welded & Experiment & 23 & $88-89$ & 129 \\
Epoxy & & 23 & 87 & 130 \\
Rigid & FEM & 23 & 90 & 131 \\
\hline
\end{tabular}


Further studies will be conducted to determine how joint parameters affect both the transmission of vibrations through the joint, and the dynamic stresses that it experiences. Other complicating phenomena such as nonlinear joint behaviour, parameter uncertainties, and localization effects will also be examined in due course.

\section{Concluding remarks}

Dynamic analysis of vehicle components and systems is now an integral part of the engineering design process in the automotive industry (Cerrato et al 1997; Roesems 1997). Various computational tools including finite and boundary element codes are employed to analyse and optimise components and sub-assemblies. The case studies presented in this article demonstrate some of the challenges that designers, analysts and experimenters face. Many fundamental issues still remain unresolved but progress is being made at several fronts.

The authors wish to thank the following students who contributed to the contents of this article: Drs. G Kim, J E Farstad, T J Royston, S W Kung and M L Liaw. The financial support from research sponsors including Teledyne Corp., Edison Welding Institute, US Army, Copeland Corp. and Chrysler Corp. is gratefully acknowledged.

\section{Refrences}

Cerrato G, Grewe A, Williams R 1997 Sound quality tools for NHV design. Sound Vibr. 31: 20-28

Farstad J E, Singh R 1995 Structurally transmitted dynamic power in discretely joined damped component assemblies. J. Acoust. Soc. Am. 97: 2855-2865

Farstad J E, Singh R 1996 Effects of modal truncation errors on transmitted dynamic power estimates in discretely joined component assemblies. J. Acoust. Soc. Am. 100: 3144-3158

Kim G, Singh R 1993 Nonlinear analysis of automotive hydraulic engine mount. J. Dyn. Syst., Meas. Contr. 115: 482-487

Kim G, Singh R 1995 A study of passive and adaptive hydraulic engine mount systems with emphasis on nonlinear characteristics. J. Sound Vibr. 179: 427-453

Roesems D 1997 A new methodology to support an optimized NVH engineering process. Sound Vibr. 31: 36-45

Rook T E, Singh R 1995 Power flow through multi-dimensional compliant joints using mobility and modal approaches. J. Acoust. Soc. Am. 97: 2882-2891

Rook T E, Singh R 1996 Modal truncation issues in synthesis procedures for vibratory power flow dissipation. J. Acoust. Soc. Am. 99:2158-2166

Rook T E, Singh R 1998 Structural intensity calculations for compliant plate-beam structures connected by bearings. J. Sound Vibr. 211: 361-387

Royston T J, Singh R 1995 Periodic response of nonlinear engine mount system. Proc. 1995 SAE Noise and Vibration Conf. pp 519-530

Royston T J, Singh R 1996 Optimization of passive and active nonlinear vibration mounting systems based on vibratory power transmission. J. Sound Vibr. 194: 295-316

Royston T J, Singh R 1997 Vibratory power flow through a nonlinear path into a resonant receiver. $J$. Acous. Soc. Am. 101: 2059-2069

Singh R, Kim G, Ravindra P V 1992 Linear analysis of an automotive hydro-mechanical mount with emphasis on decoupler characteristics. J.Sound Vibr. 158: 219-243

Singh R, Liaw M L, Farstad J E 1995 Determination of joint stiffness through vibration analysis of beam assemblies. ASME paper \#95-WA/NCA-28 